\title{
Effect of PEO/PPO Type Triblock Copolymers on Dispersion Behaviour of Aqueous Alumina Suspensions
}

\author{
Aylin M.ŞAKAR ${ }^{1}$, Hürriyet POLAT ${ }^{2}$ and Muhsin ÇİFTÇİĞLU ${ }^{3}$ \\ Izmir Institute of Technology, TURKEY
}

Key Words: Alumina, dispersion, stabilization and block copolymers.

\begin{abstract}
The aim of this study was to investigate the stability and dispersion behaviour of aqueous alumina suspensions in the presence of polyethylene oxide-polypropylene oxide-polyethylene oxide (PEO/PPO/PEO) type block copolymers.

The influence of water soluble triblock copolymers with molecular weight ranging from 2900 to $12600 \mathrm{~g} /$ mole were tested at $\Phi=0.125,1,10,20 \mathrm{vol} \%$ solid loading alumina suspensions by rheological, turbidity, sedimentation and surface tension measurements. The results indicate that an alternative dispersant for alumina suspensions is the PEO/PPO triblock copolymers. Their stabilisation mechanism was attributed to the depletion stabilisation. Rheological measurements indicated that these block copolymers (at high EO percentages) coupled with ultrasonic treatment have a positive effect on the dispersion of the agglomerated alumina suspensions. It was not possible to create stable dispersions in the absence of ultrasonic bath treatment. Turbidity measurements at $\Phi=0.025,0.125$ vol \% showed that, alumina suspensions with $\mathrm{PEO} / \mathrm{PPO}$ added were stable and have higher turbidity values than block copolymer free suspensions.
\end{abstract}

\section{Introduction}

The interaction between particles dispersed in aqueous media with each other and with the surrounding phase has a great importance for ceramic processing. The preparation of high quality ceramics necessitates the successful dispersion of powders and the preparation of stable suspensions. Alumina $\left(\mathrm{Al}_{2} \mathrm{O}_{3}\right)$ is the most widely used oxide ceramic because it is plentiful, relatively low in cost and equal to or better than most oxides in mechanical properties [1]. Alumina powders in aqueous media are normally very unstable because the fine powder particles have tendency to agglomerate. Consolidation of these powders may usually involve the use of stable suspensions. 
Polyethylene oxide-polypropylene oxide-polyethylene oxide (PEO/PPO/PEO) type block copolymers are used as dispersing agents in many industrial applications. However there is no information on the use of these polymers in alumina suspension preparation. The aim of the present work was the investigation of the effects of PEO/PPO/PEO type block copolymers and the use of ultrasonic treatment on aqueous alumina suspension properties.

\section{Experimental}

\section{Materials}

Two commercial high purity $\alpha-\mathrm{Al}_{2} \mathrm{O}_{3}$ powders were used in this study. The first powder (AKP-53) has an average particle size of $0.29 \mu \mathrm{m}$ was supplied by Sumitomo Chemicals Co., Japan. The second powder with an average particle size of $<10 \mu \mathrm{m}$ was purchased from Aldrich Chemicals Co.

$\mathrm{PEO} / \mathrm{PPO}$ triblock copolymers (Commercially known as Pluronics, F-127 with $\mathrm{Mw}=$ $12600, \mathrm{~F}-68 \mathrm{Mw}=8400, \mathrm{P}-104=5900, \mathrm{PE}-6400=2900)$ were obtained from BASF Corporation, Washington, NJ. Polyacrylic acid that was used has $\mathrm{M}_{\mathrm{W}}$ of $2000 \mathrm{~g} / \mathrm{mole}$

\section{Method}

Suspensions were prepared by using commercial powders that is AKP-53 and Aldrich alumina. Solid loadings in suspensions were adjusted as $\Phi=0.125,1,10,20$ vol $\%$ for the viscosity measurements. Suspensions for mentioned experiments were prepared by using four different methods:

- Method I: Alumina and water $\rightarrow 10$ mins. stirring.

- Method II: Alumina and water $\rightarrow 10$ mins. stirring +10 mins. ultrasonic treatment +10 mins. stirring.

- Method III : Alumina, water and surfactant $\rightarrow 10$ mins. stirring.

- Method IV: Alumina, water and surfactant $\rightarrow 10$ mins. stirring +10 mins. ultrasonic treatment +10 mins. stirring.

Rheological behaviour of the suspensions was determined by using Brookfield DV-III RV rheometer with UL adaptor. Viscosities of the suspensions were measured at different shear rates by use of the following input conditions:

- Shear rate was set at max shear $250 \mathrm{~s}^{-1}$

- It was decreased to $100 \mathrm{~s}^{-1}$ by $10 \mathrm{~s}^{-1}$ in every $30 \mathrm{sec}$.

- Shear rate was again started to increase by 10 increments up to $250 \mathrm{~s}^{-1}$.

Turbidity measurements were performed by using Hach model 2100 AN turbidimeter. Adsorption behaviour of the surfactants was studied by surface tension measurements by using a ring type Kruss- Digital Tensiometer K 10ST. 


\section{Results and Discussion}

\section{Rheological Measurements}

The effects of three different surfactants; F-127, F-68, PAA and ultrasonic treatment on alumina powder suspensions (AKP-53) at $\Phi=20$ vol $\%$ solid loading is shown in Fig.1. According to the Fig.1 (a) there is a big difference between the initial increasing rate sweep and the later decreasing rate sweep, which was assumed to be due to flocculation. Initial part of the curve has a typical shear thinning character with $1.34 \mathrm{~N} / \mathrm{m}^{2}$ yield stress. In Fig. 1 (c) at $10^{-6} \mathrm{M}$ PAA concentration, there is a disturbance for the dispersion behaviour. At $10^{-3} \mathrm{M}$ PAA there was no measurable data because of strong agglomeration. For the case of block copolymer of F-127, viscosity results of the slurry, showed that the Newtonian behaviour at $10^{-3} \mathrm{M}$ similar to $\mathrm{F}-68$.
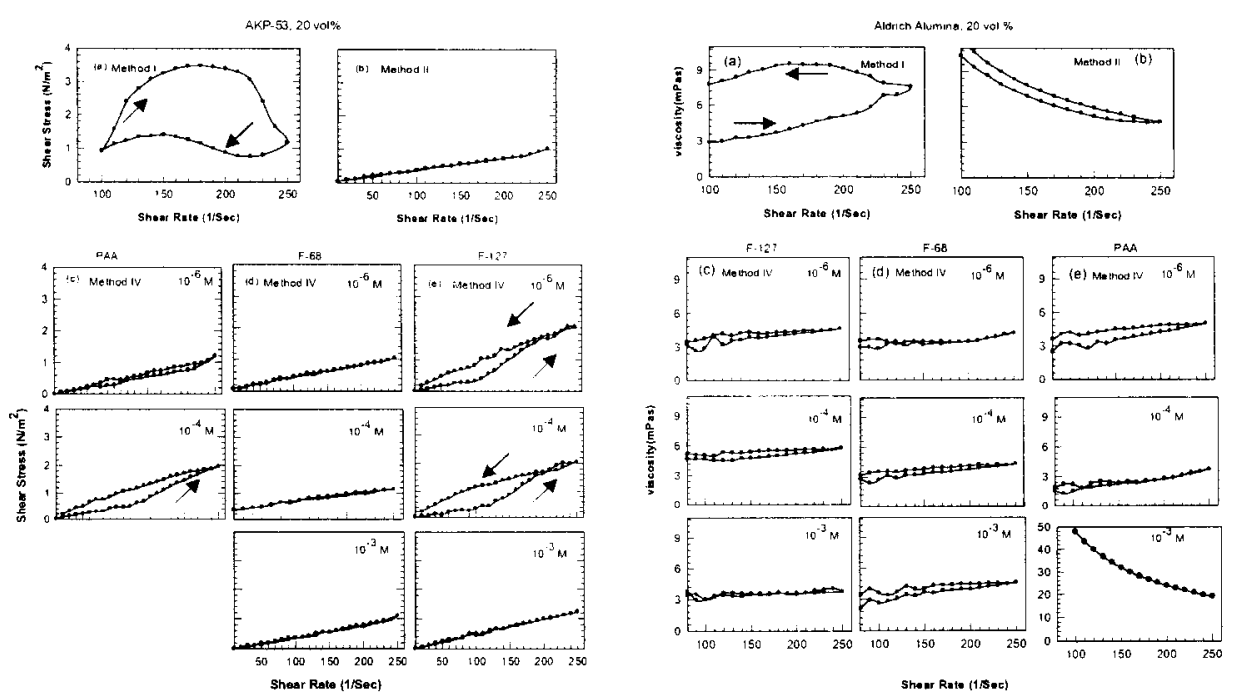

Figure 1. Shear rate vs. stress graph of AKP-53 at $20 \mathrm{vol} \%$.

Figure 2. Shear rate vs. stress graph of

Aldrich alumina at 20 vol \%.

For the Aldrich alpha alumina powder at $\Phi=20 \mathrm{vol} \%$, slurry without any treatment showed an undispersed behaviour. There is a big difference between the initial and the final rate sweeps. But the usage of ultrasonic treatment did not bring a sufficient effect to create the stable dispersion. In contrast viscosity of the suspension increased detrimentally. On the other hand F-68 and F-127 has showed an important effect to get the slurry to a dispersed form. 


\section{Sedimentation and Turbidity Measurements}

The sediment height of suspensions that was prepared with 4 different methods is shown in Fig. 3 as a function of time. AKP-53 suspensions of 20 vol \% prepared with method (III) at $10^{-2} \mathrm{M} \mathrm{F-127}$ concentration produced a clear supernatant with an extremely high sediment height. In contrast method (IV) produced more stabile suspensions. Similarly in turbidity measurements highest turbidity values $(>10000$ NTU) were obtained in the case of method (IV) with F-127.

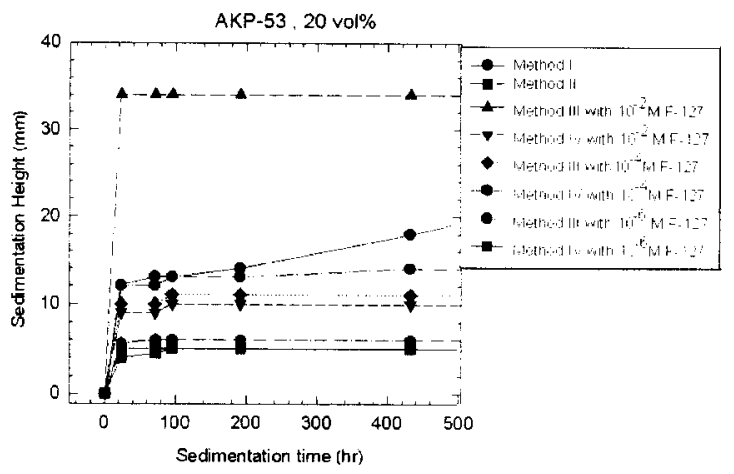

Figure 3. Sedimentation time vs. height plot of AKP-53 suspensions at 20 vol $\%$.

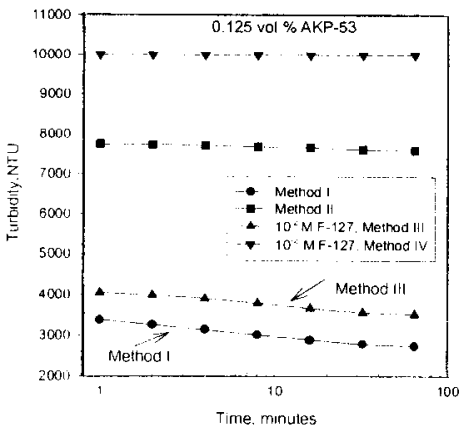

Figure 4. Time vs. turbidity graph of AKP-53 at $0.125 \mathrm{vol} \%$.

\section{Summary and Conclusions}

The effects of the blockcopolymers of $\mathrm{PEO} / \mathrm{PPO} / \mathrm{PEO}$ were investigated in this work.Results indicated that combined with ultrasonic treatment $\mathrm{PEO} / \mathrm{PPO} / \mathrm{PEO}$ were found to be capable of producing well stabilized alumina suspensions. According to the surface tension results the they did not adsorb onto the Aldrich alumina particles. This result supports the fact that depletion mechanism may be responsible from the stabilization of the alumina suspensions investigated in this work.

\section{References}

[1] Heimenz P.C.; Principles of Colloid \& Surface Chemistry, $3^{\text {rd }}$ edition, Marcel Dekker, (1997)

[2] Cesarano III J., Aksay I., "Processing of Highly Concentrated Aqueous $\alpha$-Alumina Suspensions Stabilized with Polyelectrolytes." Journal of The American Ceramic Society Vol

71 No: 12 (1988), 1062-1067 


\section{Euro Ceramics VII}

10.4028/www.scientific.net/KEM.206-213

Effect of PEO/PPO Type Triblock Copolymers on Dispersion Behaviour of Aqueous Alumina Suspensions

10.4028/www.scientific.net/KEM.206-213.421 